Rapid Reviews COVID-19

\title{
Reviews of "Short-term effect of the COVID-19 pandemic on suicidal ideation: A prospective cohort study"
}

\section{Traolach Brugha ${ }^{1}$, Chang Shu-Sen ${ }^{2}$}

${ }^{1}$ Professor of Psychiatry, University of Leicester Department of Health Sciences, Health Sciences, UK,

${ }^{2}$ National Taiwan University, Institute of Health Behaviors and Community Sciences, Taiwan

Published on: Nov 06, 2020

DOI: $10.1162 / 2 \mathrm{e} 3983 f 5 . \mathrm{a} 1605 f 23$

License: Creative Commons Attribution 4.0 International License (CC-BY 4.0). 
To read the original manuscript, click the link above.

Summary of Reviews: This is a potentially informative study about changes in rates of suicidal ideation during the pandemic, however reviewers raised concerns about the validity of the methods, statistical approaches used as well as cohort attrition rates.

\section{Reviewer 1 (Traolach Brugha)}

Reviewer 2 (Chang Shu-Sen)

$$
\begin{aligned}
& \text { RR:C19 Strength of Evidence Scale Key. }
\end{aligned}
$$

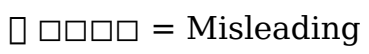

$$
\begin{aligned}
& \text { प्र } \square \square \square=\text { Not Informative } \\
& \text { प्रा पि = Potentially Informative }
\end{aligned}
$$

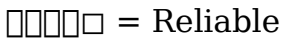

$$
\begin{aligned}
& \text { प्राप्र = Strong }
\end{aligned}
$$

To read the reviews, click the links below. 